\title{
AN ESTIMATE FOR CERTAIN MEROMORPHIC UNIVALENT FUNCTIONS
}

MINGQIN XIE

(Communicated by Irwin Kra)

ABSTRACT. In this paper the coefficient problem for the family of univalent functions

$$
g(z)=z+\sum_{n=1}^{\infty} b_{n} z^{-n}
$$

in $\{|z|>1\}$ has been studied. The author obtained the sharp estimate $b_{7} \leq$ $1 / 4+3 / 280$ when $g(z)$ is an odd function and all its coefficients are real.

Let $\Sigma_{0}$ denote the class of functions $g(z)$ univalent in $|z|>1$, regular apart from a simple pole at the point at infinity and having the expansion at that point

$$
g(z)=z+\sum_{n=1}^{\infty} b_{n} z^{-n}
$$

Garabedian and Schiffer [1] obtained the sharp estimate $\left|b_{3}\right| \leq\left(1+2 e^{-6}\right) / 2$, and at the same time they remarked that if all the coefficients $b_{n}$ of $g(z)$ are real, then $b_{3} \leq 1 / 2$. Furthermore, Kubota [2] obtained an estimate of $b_{5}$. He proved the following theorem.

THEOREM. If all the coefficients $b_{n}$ of $g(z)$ are real, then

$$
b_{5} \leq \frac{1}{3}+\frac{4}{507}
$$

with equality holding only for the function $\tilde{g}(z)$ which satisfies the algebraic equation

$$
\left(w^{2}+\frac{12}{13}\right)^{3}=\left(z^{3}+\frac{6}{13} z+\frac{6}{13} z^{-1}+z^{-3}\right)^{2}, \quad w=\tilde{g}(z) .
$$

The expansion of $\tilde{g}(z)$ at the point at infinity begins

$$
z-\frac{4}{13} z^{-1}+\frac{16}{169} z^{-3}+\left(\frac{1}{3}+\frac{4}{507}\right) z^{-5}+\cdots .
$$

$A s b_{2}=b_{4}=0$ for $\tilde{g}(z)$, it is easy to verify by induction that $\tilde{g}(z)$ is an odd function in $\Sigma_{0}$.

In this paper we shall be concerned with the coefficient $b_{7}$ and prove the following.

Received by the editors April 28, 1986 and, in revised form, October 17, 1986.

1980 Mathematics Subject Classification (1985 Revision). Primary 30A32; Secondary 30A34.

Key words and phrases. Univalent, coefficient, trajectory, quadratic differential. 
THEOREM 1. If $F(z)=z+\sum_{n=1}^{\infty} b_{2 n-1} z^{-2 n+1}$ is an odd function in $\Sigma_{0}$ and all its coefficients $b_{2 n-1}$ are reals, then

$$
b_{7} \leq \frac{1}{4}+\frac{3}{280}
$$

with equality holding only for the functions $\tilde{F}_{j}(z)$ which satisfy the algebraic equations

$$
w^{4}+t_{j} w^{2}=G_{j}(z), \quad w=\tilde{F}_{j}(z), \quad j=1,2
$$

where

$$
\begin{gathered}
t_{1}=-5 \sqrt{\frac{6}{36}}, \quad t_{2}=5 \sqrt{\frac{6}{35}}, \\
G_{1}(z)=z^{4}-\sqrt{\frac{6}{35}} z^{2}-\frac{26}{35}-\sqrt{\frac{6}{35}} z^{-2}+z^{-4}, \\
G_{2}(z)=z^{4}+\sqrt{\frac{6}{35}} z^{2}-\frac{26}{35}+\sqrt{\frac{6}{35}} z^{-2}+z^{-4} .
\end{gathered}
$$

The expansions of $\tilde{F}_{j}(z)$ at the point at infinity begin

$$
\begin{aligned}
& \tilde{F}_{1}(z)=z+\sqrt{\frac{6}{35}} z^{-1}-\frac{1}{70} z^{-3}-\frac{1}{5} \sqrt{\frac{6}{35}} z^{-5}+\left(\frac{1}{4}+\frac{3}{280}\right) z^{-7}+\cdots \\
& \tilde{F}_{2}(z)=z-\sqrt{\frac{6}{35}} z^{-1}-\frac{1}{70} z^{-3}+\frac{1}{5} \sqrt{\frac{6}{35}} z^{-5}+\left(\frac{1}{4}+\frac{3}{280}\right) z^{-7}+\cdots
\end{aligned}
$$

Our proof is due to Goluzin's inequality.

Firstly we give two lemmas which will be used later.

Let $\sigma_{m}$ denote the class of all analytic and $m$-valent functions for $|z|>1$ with a development

$$
F(z)=\sum_{n=-m}^{\infty} b_{n} z^{-n}, \quad b_{-m} \neq 0 .
$$

LEMMA 1 [3]. Suppose $F \in \sigma_{m}$, and $P_{q}(w)=\sum_{n=0}^{q} a_{n} w^{n}$ is an arbitrary nonconstant polynomial of degree $q$. Then $P_{q}(F(z)) \in \sigma_{m q}$.

LEMMA 2 [4]. If $F \in \sigma_{m}$ with the development (2), then

$$
\sum_{n=1}^{\infty} n\left|b_{n}\right|^{2} \leq \sum_{n=1}^{m} n\left|b_{-n}\right|^{2} .
$$

The inequalities that arise from (3) are known as the Goluzin inequalities.

Now we shall prove Theorem 1. Set

$$
\begin{aligned}
& F(z)^{2}=z^{2}+\sum_{n=0}^{\infty} c_{2 n} z^{-2 n} \\
& F(z)^{4}=z^{4}+\sum_{n=-1}^{\infty} d_{2 n} z^{-2 n} .
\end{aligned}
$$

Obviously, in our case all coefficients $c_{2 n}$ and $d_{2 n}$ are real. The relations between the coefficients are

$$
\begin{aligned}
& c_{0}=2 b_{1} \\
& c_{2}=b_{1}^{2}+2 b_{3} \\
& c_{4}=2 b_{1} b_{3}+2 b_{5} \\
& c_{6}=b_{3}^{2}+2 b_{1} b_{5}+2 b_{7}
\end{aligned}
$$


and

$$
\begin{aligned}
& d_{-2}=2 c_{0}, \\
& d_{0}=c_{0}^{2}+2 c_{2}, \\
& d_{2}=2 c_{0} c_{2}+2 c_{4}, \\
& d_{4}=c_{2}^{2}+2 c_{0} c_{4}+2 c_{6} .
\end{aligned}
$$

We introduce a real parameter $t$ and apply Lemma 2 to the function $F(z)^{4}+$ $t F(z)^{2}$, which is 4-valent with a pole of order four at infinity. This gives

$$
\left(c_{2} t+d_{2}\right)^{2}+2\left(c_{4} t+d_{4}\right)^{2} \leq\left(t+d_{-2}\right)^{2}+2 .
$$

After rearrangement, (7) becomes

$$
\left(1-c_{2}^{2}-2 c_{4}^{2}\right) t^{2}+2\left(d_{-2}-c_{2} d_{2}-2 c_{4} d_{4}\right) t+2+d_{-2}^{2}-d_{2}^{2}-2 d_{4}^{2} \geq 0 .
$$

Applying Lemma 2 to the function $F(z)^{2}$ which belongs to $\sigma_{2}$, we find that the coefficient of $t^{2}$ in (7) is nonnegative. The positive definiteness of the Hermitian form implies

$$
\left(d_{-2}-c_{2} d_{2}-2 c_{4} d_{4}\right)^{2} \leq\left(1-c_{2}^{2}-2 c_{4}^{2}\right)\left(2+d_{-2}^{2}-d_{2}^{2}-2 d_{4}^{2}\right) .
$$

We shall show that $d_{4}$ lies inside a certain circle:

$$
\left|d_{4}-\omega\right| \leq R \text {. }
$$

To do so we bring (8) into the form

$$
\begin{aligned}
{\left[d_{4}-\frac{c_{4}\left(d_{-2}-c_{2} d_{2}\right)}{1-c_{2}^{2}}\right]^{2} \leq } & \frac{\left(1-c_{2}^{2}-2 c_{4}^{2}\right)\left(2+d_{-2}^{2}-d_{2}^{2}\right)}{2\left(1-c_{2}^{2}\right)} \\
& -\frac{\left(d_{-2}-c_{2} d_{2}\right)^{2}}{2\left(1-c_{2}^{2}\right)}+\frac{c_{4}^{2}\left(d_{-2}-c_{2} d_{2}\right)^{2}}{\left(1-c_{2}^{2}\right)^{2}}
\end{aligned}
$$

With the aid of (6) we thus have

$$
\omega=2 c_{0} c_{4}-\frac{2 c_{2} c_{4}^{2}}{1-c_{2}^{2}}
$$

and upon using the identity

$$
\left(d_{-2}-c_{2} d_{2}\right)^{2}-\left(c_{2} d_{-2}-d_{2}\right)^{2}=\left(d_{-2}^{2}-d_{2}^{2}\right)\left(1-c_{2}^{2}\right),
$$

it turns out that $R$ has the value

$$
R=\frac{1-c_{2}^{2}-2 c_{4}^{2}}{1-c_{2}^{2}}
$$

From (6), (10), and (11), inequality (9) implies

$$
c_{6} \leq \frac{1}{2}-\frac{1}{2} c_{2}^{2}-\frac{c_{4}^{2}}{1-c_{2}} .
$$

Substituting the relations (5), we find that (12) becomes

$$
b_{7} \leq \frac{1}{4}+\Phi\left(b_{1}, b_{3}, b_{5}\right)
$$

where

$$
\Phi\left(b_{1}, b_{3}, b_{5}\right)=-\frac{1}{2} b_{3}^{2}-\frac{1}{4}\left(b_{1}^{2}+2 b_{3}\right)^{2}-b_{1} b_{5}-\frac{2\left(b_{1} b_{3}+b_{5}\right)^{2}}{1-b_{1}^{2}-2 b_{3}}
$$


Simple computing shows that the function $\Phi\left(b_{1}, b_{3}, b_{5}\right)$ achieves its maximum at the points

$$
P_{1}\left(\sqrt{\frac{6}{35}},-\frac{1}{70},-\frac{1}{5} \sqrt{\frac{6}{35}}\right) \text { and } P_{2}\left(-\sqrt{\frac{6}{35}},-\frac{1}{70}, \frac{1}{5} \sqrt{\frac{6}{35}}\right) .
$$

Hence we have

$$
\begin{aligned}
b_{7} & \leq \frac{1}{4}+\Phi\left(\sqrt{\frac{6}{35}},-\frac{1}{70},-\frac{1}{5} \sqrt{\frac{6}{35}}\right) \\
& =\frac{1}{4}+\Phi\left(-\sqrt{\frac{6}{35}},-\frac{1}{70}, \frac{1}{5} \sqrt{\frac{6}{35}}\right)=\frac{1}{4}+\frac{3}{280} .
\end{aligned}
$$

Next, the equality occurs only for

$$
\left(b_{1}, b_{3}, b_{5}\right)=P_{j}, \quad j=1,2,
$$

and

$$
d_{4}=\omega+R .
$$

In this case the equality of (7) holds for some $t=t_{j}$ and

$$
c_{2 n} t_{j}+d_{2 n}=0, \quad n \geq 3, j=1,2 .
$$

Substitute (5), (6), (14), and (15) in (7). We find

$$
\begin{array}{cc}
t_{1}=-5 \sqrt{\frac{6}{35}}, & t_{2}=5 \sqrt{\frac{6}{35}}, \\
t_{1}+d_{-2}=-\sqrt{\frac{6}{35}}, & t_{2}+d_{-2}=\sqrt{\frac{6}{35}}, \\
c_{0} t_{1}+d_{0}=-\frac{26}{35}, & c_{0} t_{2}+d_{0}=-\frac{26}{35}, \\
c_{2} t_{1}+d_{2}=-\sqrt{\frac{6}{35}}, & c_{2} t_{2}+d_{2}=\sqrt{\frac{6}{35}}, \\
c_{4} t_{1}+d_{4}=1, & c_{4} t_{2}+d_{4}=1 .
\end{array}
$$

Thus (1) is true.

Finally, we shall prove that the $\tilde{F}_{j}(z)(j=1,2)$ belong to $\Sigma_{0}$.

Let $Q^{*}\left(w ; t_{j}\right) d w^{2}(j=1,2)$ be the quadratic differential

$$
w^{2}\left(w^{2}+\frac{1}{2} t_{j}\right)^{2} d w^{2}, \quad j=1,2 .
$$

For each value of $j$ there are four end domains $E_{1 j}^{*}, E_{2 j}^{*}, E_{3 j}^{*}, E_{4 j}^{*}$, in the trajectory structure of $Q^{*}\left(w ; t_{j}\right) d w^{2}$ on the upper half $w$-plane. For a suitable determination each of the functions

$$
\varsigma=\int w\left(w^{2}+\frac{1}{2} t_{j}\right) d w, \quad j=1,2,
$$

maps $E_{1 j}^{*}, E_{2 j}^{*}, E_{3 j}^{*}, E_{4 j}^{*}$ respectively onto an upper half-plane, a lower half-plane, an upper half-plane and a lower half-plane, the positive real axis, corresponding to the half-infinite segment $\operatorname{Im} \varsigma=0, \frac{1}{16} t_{j}^{2}<\operatorname{Re} \varsigma<\infty$.

On the other hand, for each value of $j$ there are four end domains $E_{1 j}, E_{2 j}, E_{3 j}$, $E_{4 j}$ in the trajectory structure of the quadratic differential

$$
\begin{aligned}
& z^{-10}(z-1)^{2}(z+1)^{2}(z-i)^{2}(z+i)^{2}\left(z-e^{i \theta_{j}}\right)^{2} \\
& \cdot\left(z+e^{i \theta_{j}}\right)^{2}\left(z-e^{-i \theta_{j}}\right)^{2}\left(z+e^{-i \theta_{j}}\right)^{2} d z^{2}, \quad j=1,2,
\end{aligned}
$$




$$
\cos 2 \theta_{1}=\frac{1}{4} \sqrt{\frac{6}{35}}, \quad \cos 2 \theta_{2}=-\frac{1}{4} \sqrt{\frac{6}{35}},
$$

on the domain $|z|>1, \operatorname{Im} z>0$. For a suitable determination each of the functions

$$
\begin{aligned}
\varsigma= & \int z^{-5}(z-1)(z+1)(z-i)(z+i)\left(z-e^{i \theta_{j}}\right) \\
& \cdot\left(z+e^{i \theta_{j}}\right)\left(z-e^{-i \theta_{j}}\right)\left(z+e^{-i \theta_{j}}\right) d z, \quad j=1,2,
\end{aligned}
$$

maps $E_{1 j}, E_{2 j}, E_{3 j}, E_{4 j}$ respectively onto an upper half-plane, a lower half-plane, an upper half-plane and a lower half-plane, the points $1, e^{i \theta_{j}}, i,-e^{-i \theta_{j}},-1$ corresponding to the points

$$
\begin{gathered}
\frac{1}{2}-2 \cos 2 \theta_{j}+\frac{23}{280}, \quad-\frac{1}{2}-\cos ^{2} 2 \theta_{j}+\frac{23}{280}, \\
\frac{1}{2}+2 \cos 2 \theta_{j}+\frac{23}{280}, \quad-\frac{1}{2}-\cos ^{2} 2 \theta_{j}+\frac{23}{280}, \quad \frac{1}{2}-2 \cos 2 \theta_{j}+\frac{23}{280} .
\end{gathered}
$$

Thus we can combine functions (16) with (17) respectively for $j=1,2$ to obtain two functions, each of which maps the domain $|z|>1, \operatorname{Im} z>0$ into the upper half $w$-plane. By reflection these functions extend to the functions $\tilde{F}_{j}(z)(j=$ 1,2) respectively, which map $|z|>1$ onto a domain admissible with respect to $Q^{*}\left(w ; t_{j}\right) d w^{2}(j=1,2)$. It means that $\tilde{F}_{j}(z)(j=1,2)$ belong to $\Sigma_{0}$. This completes the proof of Theorem 1 .

\section{REFERENCES}

1. P. R. Garabedian and M. Schiffer, A coefficient inequality for schlicht functions, Ann. of Math. (2) 61 (1955), 116-136.

2. Y. Kubota, $A$ coefficient inequality for certain meromorphic univalent functions, Kodai Math. Sem. Rep. 26 (1974), 85-94.

3. Н. А. Лебедев, Принцип площадей в теории однолистных функции, "Nauka", Moscow, 1975 , pp. 86-88.

4. L. V. Ahlfors, Conformal invariants, McGraw-Hill, 1972, pp. 86-87.

Department of Mathematics, Anhui Normal University, Wuhu, Anhui ProvINCE, PEOPLE'S REPUBLIC OF CHINA 